\title{
Observing dynamics of transparent samples by harmonically matched grating-based full-field quadrature phase interferometer
}

Jigang Wu, Zahid Yaqoob, Xin Heng, Lap Man Lee, Xiquan Cui, et al.

Jigang Wu, Zahid Yaqoob, Xin Heng, Lap Man Lee, Xiquan Cui, Changhuei Yang, "Observing dynamics of transparent samples by harmonically matched grating-based full-field quadrature phase interferometer," Proc. SPIE 6861, Three-Dimensional and Multidimensional Microscopy: Image Acquisition and Processing XV, 686102 (20 February 2008); doi: $10.1117 / 12.759210$

SPIE. Event: SPIE BiOS, 2008, San Jose, California, United States 


\title{
Observing dynamics of transparent samples by harmonically matched grating-based full-field quadrature phase interferometer
}

\author{
Jigang $\mathrm{Wu}^{*}$, Zahid Yaqoob, Xin Heng, Lap Man Lee, Xiquan Cui, Changhuei Yang \\ Biophotonics Laboratory, Department of Electrical Engineering \\ California Institute of Technology, Pasadena, CA, 91125
}

\begin{abstract}
Our group has reported the use of harmonically matched diffraction grating for full-field quantitative phase imaging. In this paper, we show the improvement of this technique and the application in observing dynamics of transparent samples. By using the grating as a beam splitter/combiner in an interferometer, we are able to obtain non-trivial phase difference between the output ports of the grating. We have built a Mach-Zehnder interferometer using the holographic grating with 600 and 1200 lines $/ \mathrm{mm}$ spacing. Two CCD cameras at the output ports of the grating-based Mach-Zehnder interferometer are used to record the full-field quadrature interferograms, which are subsequently processed to reconstruct the phase image. Since the two quadrature interferograms are acquired at the same time, the imaging speed of the system is limited only by the frame rate of the CCD cameras. We have demonstrated the capability of our system by observing dynamics of transparent samples.
\end{abstract}

Keywords: Diffraction gratings, quadrature phase interferometry, medical and biological imaging

\section{INTRODUCTION}

Phase imaging techniques such as phase contrast microscopy ${ }^{1,2}$ and Nomarski differential interference contrast (DIC) microscopy ${ }^{3}$ have been developed and widely used in biological studies. However, these techniques can only obtained qualitative phase information, which limit their application. Full-field quantitative phase imaging techniques is very important for studying biological processes especially for transparent samples such as living cells. Last few years has witnessed the development of many such techniques ${ }^{4-14}$. The imaging speeds of some techniques are fast enough for studying dynamics of transparent samples ${ }^{11,12}$. However, these techniques generally require some kind of complexity. For example, the phase shifting interferometry ${ }^{4-6}$ needs three or four interferograms with precise phase shift between them; Digital holography ${ }^{7-8}$ or Hilbert phase microscopy ${ }^{9}$ require high spatial fringes in the interferogram, which limit their field of view; Swept-source phase microscopy require the special swept laser source ${ }^{10}$.

Previously, we have developed a full-field phase imaging technique based on a harmonically matched diffraction grating pair (G1G2 grating) ${ }^{15,16}$. The grating is used as a beam splitter in a Mach-Zehnder interferometer to confer nontrivial phase shift between the two output ports of the interferometer. The two output interferograms are then recorded by two CCD cameras and processed to reconstruct the amplitude and phase image of the sample. This technique provides a simple way to implement phase microscopy compared with others.

In this paper, we report the improvement of this technique. Compared with the previous system, we have made improvement in two issues: First, we improve the optical setup to remove the geometric aberration introduced by the grating in the previous setup and get a better image quality. Second, we improve the data processing algorithm and remove the requirement in the previous system where the reference power had to be much greater than the sample. This gives the new system a better dynamic range.

\footnotetext{
*jigang@caltech.edu; phone 16263954711
}

Three-Dimensional and Multidimensional Microscopy: Image Acquisition and Processing XV

edited by Jose-Angel Conchello, Carol J. Cogswell, Tony Wilson, Thomas G. Brown

Proc. of SPIE Vol. 6861, 686102, (2008) · 1605-7422/08/\$18 - doi: 10.1117/12.759210 


\section{INTRODUCTION OF THE G1G2 GRATING}

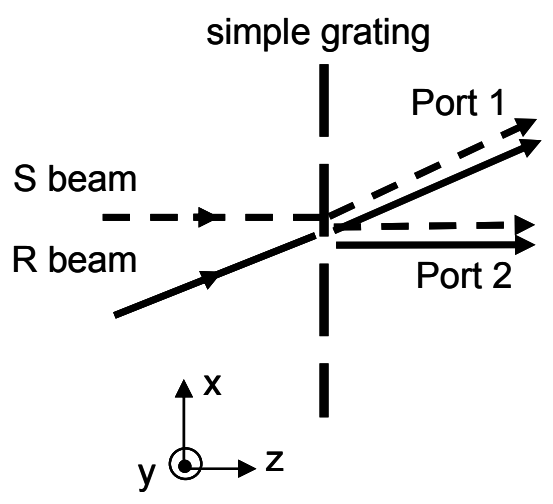

(a)

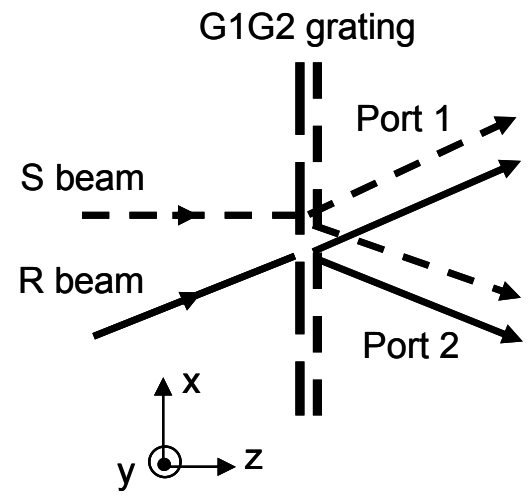

(b)

Fig. 1. (a) Simple grating in an interferometer setup; the phase difference between the interference signals in port 1 and 2 will be $180^{\circ}$; (b) G1G2 grating in an interferometer setup; the interference signals in port 1 and 2 can have a non-trivial phase difference. $\mathrm{R}$ beam: reference beam; $\mathrm{S}$ beam: sample beam;

As is well known, if a conventional beam splitter is used in an interferometer setup, the phase shift between the interference signals of the two output ports will be $180^{\circ}$. So this kind of interferometer can not be used to measure phase information without resort to phase-shifting techniques or spatial fringes encoding etc.. To obtain non-trivial phase shift between the interference signals, we notice that multi-port beam splitter such as $3 \times 3$ fiber coupler ${ }^{17,18}$ or a diffraction grating $^{19}$ features different phase shifts in the outputs for the same input. However, if a simple grating is used in an interferometer setup, as shown in Fig. 1(a), the phase shift between the interference signals in port 1 and 2 will still be $180^{\circ}$. This is because for a simple shallow grating the relative phase shift of the $m^{\text {th }}$ diffracted order with respect to the zeroth order is given by ${ }^{16}$ :

$$
\phi\left(x_{0}\right)=\left\{\begin{array}{cl}
m\left[\frac{2 \pi x_{0}}{\Lambda}+\frac{\pi}{2}\right], & m \geq 1 \\
|m|\left[-\frac{2 \pi x_{0}}{\Lambda}+\frac{\pi}{2}\right], & m \leq-1
\end{array}\right.
$$

where $x_{0}$ is the displacement of the grating from some predefined origin (the coordinate system is shown in fig.1), and

$\Lambda$ is the period of the grating. According to the phase shift equation (1), we can derive an $180^{\circ}$ phase shift in port 1 and 2 in Fig. 1(a). The details were shown in Ref. 16.

If we use a harmonically matched grating pair (G1G2 grating) instead of a simple grating, as shown in Fig. 1(b), we are able to obtain non-trivial phase shift between the interference signals of the output ports 1 and 2 . The grating pair consists of two gratings G1, G2 with periods $\Lambda_{1}, \Lambda_{2}$ that satisfy $\Lambda_{1}=2 \Lambda_{2}$. The equation (1) can then be applied to G1 and G2 grating independently and the phase shift between the interference signals of output ports 1 and 2 can be calculated as ${ }^{15}$ :

$$
\Delta \phi=\frac{4 \pi\left(x_{2}-x_{1}\right)}{\Lambda_{1}}-\frac{\pi}{2}
$$

The grating pair was fabricated by three-beam interference on a holographic plate. By adjusting the relative displacement $\left(x_{2}-x_{1}\right)$ between the G1 and G2 grating during fabrication, we can set the phase shift $\Delta \phi$ to the desired nontrivial value other than $0^{\circ}$ or $180^{\circ}$. 


\section{EXPERIMENT METHOD AND DATA PROCESSING}

The experimental setup is shown in Fig. 2. The wavelength of the HeNe laser was $632.8 \mathrm{~nm}$. Objective 1 and pinhole served as a spatial filter to clean the laser beam. The focal lengths of lens $2-4$ were all $200 \mathrm{~mm}$. The microscope systems consisting objective 2 and lens 3 (lens 4) imaged the sample into the two CCDs. The G1, G2 gratings are of 600 and 1200 lines $/ \mathrm{mm}$. The phase shift $\Delta \phi$ was measured to be $92^{\circ} \pm 8^{\circ}$.

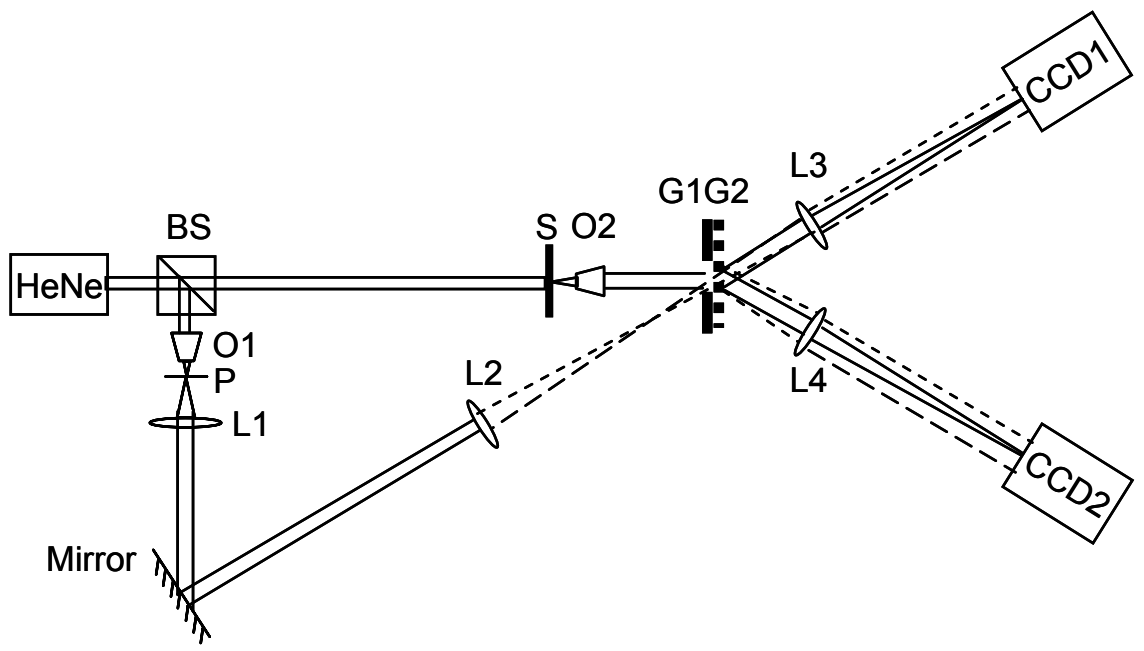

Fig. 2. Experimental setup for phase imaging. BS: beam splitter; $\mathrm{O} 1$ and O2: objective lenses 1 and 2; P: pinhole; L1-4: lens 1-4; S: sample; G1G2: the harmonically matched grating pair (G1G2 grating) on a holographic plate.

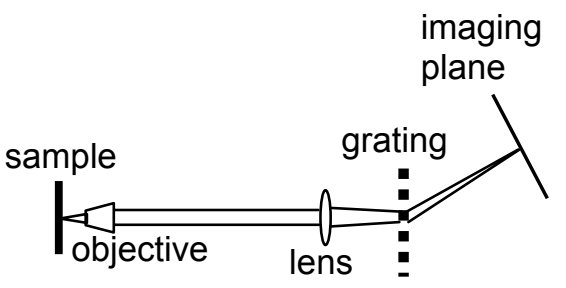

(a)

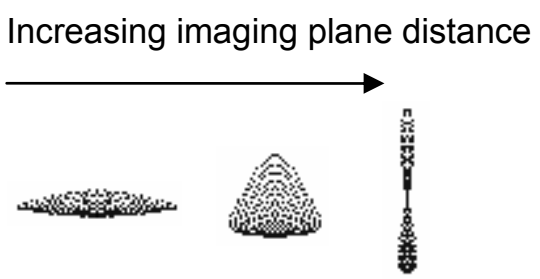

(b

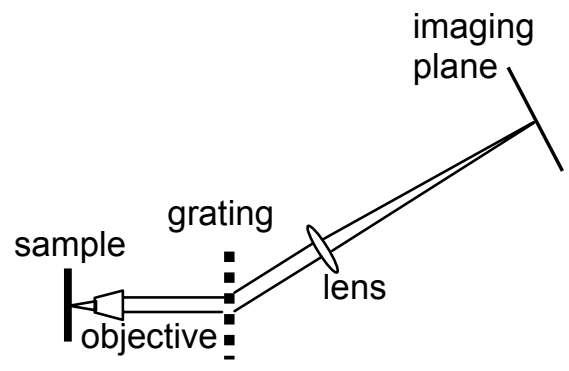

(c)

Fig. 3. Geometric aberration induced by the grating. (a) Previous imaging setup; (b) Astigmatism of the focal spots of previous setup simulated by ZEMAX; (c) Current imaging setup. 
Compared with the previous setup, the geometric aberration caused by the grating diffraction has been removed, as shown in Fig. 3. Figure 3(a) shows the imaging system used in the previous system, where in the imaging process, the incident beam at the grating originated from a sample point is converged. Since the diffraction angle of the grating is related to the sine of the incident beam angle instead of a linear dependence, we would expect to see some aberrations in the image. The simulation by the optics software ZEMAX confirmed our expectation, as shown in Fig. 3(b). As we increase the imaging plane distance, we observe the change of focuses for an object point. The aberration is similar to astigmatism which is common in optical systems.

In the current system, the imaging system is shown in Fig. 3(c), where the incident beam at the grating originated from a sample point is collimated, and thus there is no aberration caused by the diffraction of the grating. And the remained aberration in the system is due to the objective and the lens, which is very small for good optical components.

Another improvement to the previous system is the data processing algorithm. The detailed data processing procedure is explained as follows.

The detected power in corresponding pixels of the CCDs can be written as (see Fig. 2)

$$
\begin{aligned}
& P_{1}=P_{r 1}+P_{s 1}+2 \sqrt{P_{r 1} P_{s 1}} \cos (\Delta \psi) \\
& P_{2}=P_{r 2}+\eta P_{s 1}+2 \sqrt{P_{r 2} \eta P_{s 1}} \cos (\Delta \psi+\Delta \phi)
\end{aligned}
$$

where $P_{r 1}$ and $P_{r 2}$ are the reference powers at the pixels of CCD1 and CCD2, respectively; $P_{s 1}$ and $P_{s 2}$ are the sample powers at corresponding pixels of the CCDs; $\eta=P_{s 2} / P_{s 1}$ is the relative diffraction efficiency of the grating for the sample beam; $\Delta \psi=\psi_{o b j}+\psi_{b k g}$, where $\psi_{o b j}$ is the optical phase change because of the sample, $\psi_{b k g}$ is the background phase of the reference beam which is due to the residue phase aberations; $\Delta \phi$ is the non-trivial phase shift of the system.

Before the experiment, the relative diffraction efficiency $\eta$, the reference power $P_{r l}$ and $P_{r 2}$, the background phase $\psi_{b k g}$, and the non-trivial phase shift $\Delta \phi$ can be calibrated. The way to calibrate $\psi_{b k g}$ and $\Delta \phi$ are to take $\mathrm{N}$ (we usually set $\mathrm{N}=100$ ) frame pairs of the interferograms without any sample. For any of the two pixels $p, q$ of the CCDs, we can get two time series of the pixel value $p(\mathrm{n})$ and $q(\mathrm{n}), \mathrm{n}=1 \ldots \mathrm{N}$. For different $\mathrm{n}$, there is a different random phase introduced by environmental disturbance. If we plot $p(\mathrm{n})$ versus $q(\mathrm{n})$, we will get an ellipse, and the shape of the ellipse is determined by the phase shift of the interference signals of the two pixels. By performing elliptic fitting ${ }^{20}$ between $p(\mathrm{n})$ and $q(\mathrm{n})$, we can measure this phase shift. Thus we can get $\psi_{b k g}$ by performing elliptic fitting between the pixels in the same CCD and get $\Delta \phi$ by performing elliptic fitting between corresponding pixels from the two CCDs.

Now we can acquire phase images of the sample by acquiring the frame pair from the two CCDs. The detected signals of corresponding pixels will satisfy equations (3) and (4). We can solve the equations for the amplitude $P_{s l}$ and phase $\psi_{o b j}$ of the sample. Canceling $\Delta \psi$ from equation (3), (4), we can get a quadratic equation for $P_{s l}$ :

$$
\begin{aligned}
{\left[\frac{1}{P_{r 1}}+\frac{\eta}{P_{r 2}}-\frac{2 \cos (\Delta \phi) \sqrt{\eta}}{\sqrt{P_{r 1} P_{r 2}}}\right] P_{s 1}{ }^{2}-} & {\left[2 \frac{P_{1}-P_{r 1}}{P_{r 1}}+2 \frac{P_{2}-P_{r 2}}{P_{r 2}}-2 \cos (\Delta \phi) \frac{\left(P_{1}-P_{r 1}\right) \eta+\left(P_{2}-P_{r 2}\right)}{\sqrt{\eta P_{r 1} P_{r 2}}}+4 \sin ^{2}(\Delta \phi)\right] P_{s 1} } \\
+ & {\left[\frac{\left(P_{1}-P_{r 1}\right)^{2}}{P_{r 1}}+\frac{\left(P_{2}-P_{r 2}\right)^{2}}{P_{r 2} \eta}-2 \cos (\Delta \phi) \frac{\left(P_{1}-P_{r 1}\right)\left(P_{2}-P_{r 2}\right)}{\sqrt{\eta P_{r 1} P_{r 2}}}\right]=0 }
\end{aligned}
$$

We will get two solutions for $P_{s l}$ from the above equation. It can be proved that if the real $P_{s l}$ satisfied the following condition, we can always use the smaller solution of equation as our solution:

$$
\frac{P_{s 1}}{P_{r 1}}+\frac{P_{s 2}}{P_{r 2}}-2 \sqrt{\frac{P_{s 1}}{P_{r 1}} \frac{P_{s 2}}{P_{r 2}}} \cos (\Delta \phi)<\sin ^{2}(\Delta \phi)
$$

For the quadrature phase shift, $\Delta \phi=90^{\circ}$, the above condition becomes

$$
\frac{P_{s 1}}{P_{r 1}}+\frac{P_{s 2}}{P_{r 2}}<1
$$


We note that this condition is less strict compared with the requirement of the previous system, where we require the sample power to be much less than the reference power. Thus, the dynamic range of the current system is improved. The solution for the sample power $P_{s l}$ can then be substituted into equation (3) and (4) to get the phase term $\Delta \psi$, and thus $\psi_{o b j}=\Delta \psi-\psi_{b k g}$. In the final wrapped phase image, we use the Flynn's minimum discontinuity algorithm ${ }^{21}$ to unwrap the image.

Note that after the calibration, the imaging speed is limited only by the speed of CCD camera.

\section{IMAGING RESULT}

We first perform phase imaging of a phase object, which has a "CIT" logo written on a polymethyl methacrylate (PMMA) layer with thickness $100 \mathrm{~nm}$. We can see in Fig. 4(a) that the intensity image has little contrast. However, the phase image shown in Fig. 4(b) has much better contrast. Fig. 4(c) shows the measured height of a step, where the thickness of the logo is $\sim 110 \mathrm{~nm}$, close to the expected value of $100 \mathrm{~nm}$.

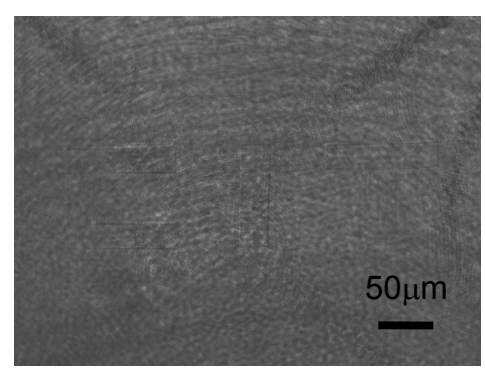

(a)

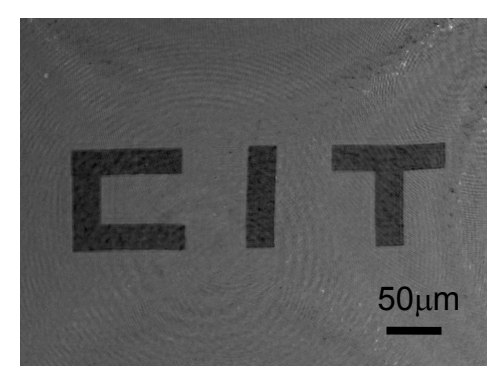

(b)

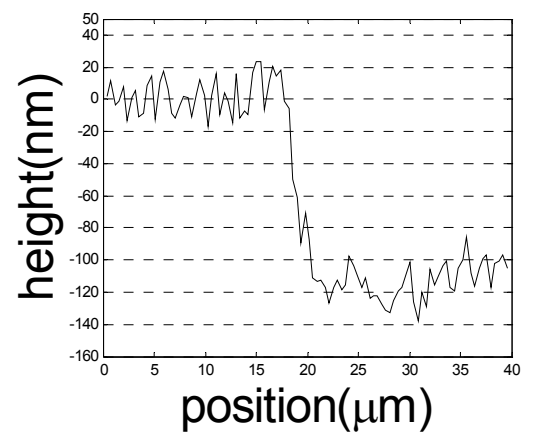

(c)

Fig. 4. Images of "CIT" logo by our iamging system. (a) intensity image; (b) phase image; (c) stepheight measurement.

We next use our system to image onion skin cell, as shown in Fig. 5. The intensity, phase images are shown in the figure. Again, the phase image has much better contrast than the intensity image. Some cell nuclei can be clearly discerned. 


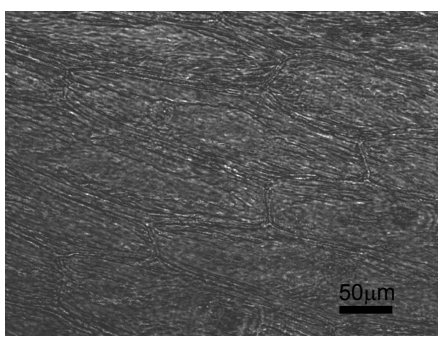

(a)

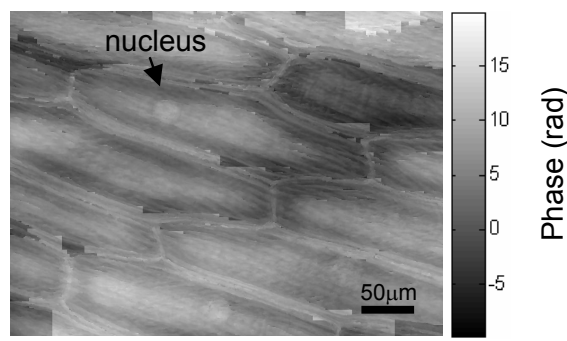

(b)

Fig. 5. Phase image of onion skin cells. (a) intensity image; (b) phase image.

Finally we applied our system to observe the movement of a paramecium caudatum. Figure 6 shows a moving paramecium caudatum at six different times with 0.05 second separation. In the figure we can see features and the movement of the paramecium.
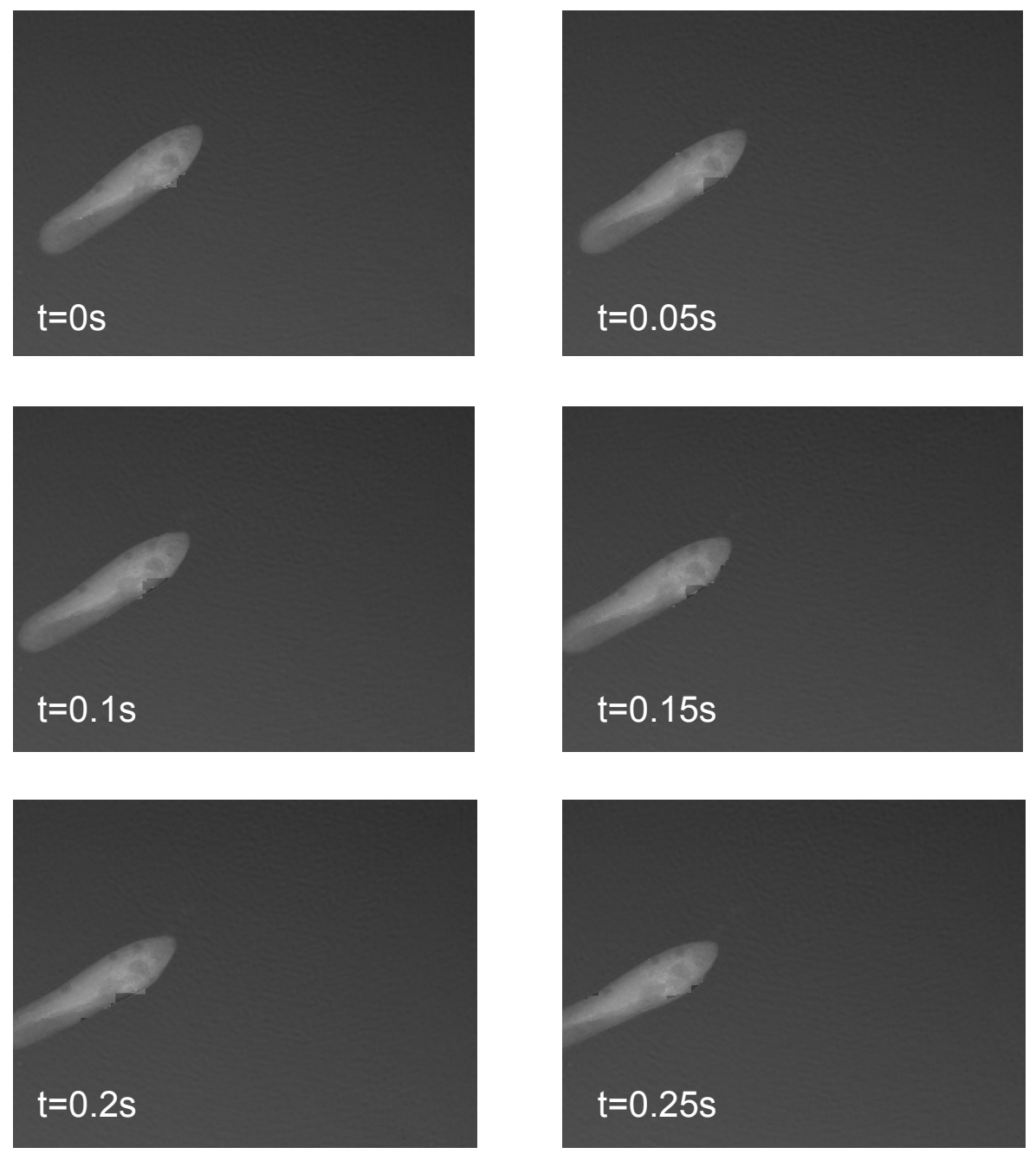

Fig. 6. Phase images of a moving paramecium caudatum at six different times with 0.05 second separation. 


\section{CONCLUSION}

We have developed an improved harmonically matched grating-based full-field quantitative phase interferometer. To demonstrate the capability of the technique, it has been applied to image pure phase object, onion skin cells, and the movement of paramecium caudatum. In the system setup, the two CCD interferograms needed to reconstruct the phase image are acquired simultaneously, which enable the system to observe fast dynamics of the sample and the imaging speed is limited only by the frame rate of the CCD. Furthermore, the G1G2 grating is a planar device that can be easily designed and fabricated in a microstructure. So it's possible to make the system more compact and flexible. In addition, the idea of using G1G2 grating can possibly be applied to other wavelengths, such as X-ray imaging because of the same basic principle.

Compared with our previous system, we have removed the geometric aberration caused by the grating, and improved the dynamic range of the system by improving the data processing algorithm. In conclusion, this technique is very useful for observing dynamics of transparent samples, and can be applied to biological studies.

\section{REFERENCES}

1. F. Zernike, "Phase contrast, a new method for the microscopic observation of transparent objects", Physica 9, 686698 (1942).

2. F. Zernike, "Phase contrast, a new method for the microscopic observation of transparent objects Part II", Physica 9, 974-986 (1942).

3. R. D. Allen, G. B. David, and G. Nomarski, "The Zeiss-Nomarski differential interference equipment for transmitted light microscopy," Z. wiss. Mikr. 69, 193-221 (1969).

4. K. Creath, in Progess in Optics, Vol. XXVI, E. Wolf, ed. (Elsevier, Amsterdam, 1988), pp349-393.

5. K. J. Chalut, W. J. Brown, and A. Wax, "Quantitative phase microscopy with asynchronous digital holography," Opt. Lett. 15, 3047-3052 (2007).

6. W. Choi, C. Fang-Yen, K. Badizadegan, S. Oh, N. Lue, R. R. Dasari, and M. S. Feld, "Tomographyic phase microscopy," Nature methods 4, 717-719 (2007).

7. P. Marquet, B. Rappaz, P. J. Magistretti, E. Cuche, Y. Emery, T. Colomb, and C. Depeursinge, "Digital holographic microscopy: a noninvasive contrast imaging technique allowing quantitative visualization of living cells with subwavelength axial accuracy," Opt. Lett. 30, 468-470 (2005).

8. J. Kuhn, T. Colomb, F. Montfort, F. Charriere, Y. Emery, E. Cuche, P. Marquet, and C. Depeursinge, "Real-time dual-wavelength digital holographic microscopy with a single hologram acquisition," Opt. Express 15, 7231-7242 (2007).

9. T. Ikeda, G. Popescu, R. R. Dasari, and M. S. Feld, "Hilbert phase microscopy for investigating fast dynamics in transparent systems," Opt. Lett. $30,1165-1167$ (2005).

10. M. V. Sarunic, S. Weinberg, and J. A. Izatt., "Full-field swept-source phase microscopy," Opt. Lett. 31, 1462-1464 (2006).

11. B. Rappaz, P. Marquet, E. Cuche, Y. Emery, C. Depeursinge, and P. Magistretti, "Measurement of the integral refractive index and dynamic cell morphometry of living cells with digital holographic microscopy," Opt. Express 13, 9361-9373 (2005).

12. G. Popescu, T. Ikeda, K. Goda, C. A. Best-Popescu, M. Laposata, S. Manley, R. R. Dasari, K. Badizadegan, and M. S. Feld, "Optical measurement of cell membrane tension," Phys. Rev. Lett. 97, 218101 (2006).

13. D. O. Hogenboom, C. A. DiMarzio. T. J. Gaudette, A. J. Devaney, and S. C. Lindberg, "Three-dimensional images generated by quadrature interferometry," Opt. Lett. 23, 783-785 (1998).

14. D. Paganin and K. A. Nugent, "Noninterferometric phase imaging with partially coherent light," Phys. Rev. Lett. 80, $2586(1998)$

15. J. Wu, Z. Yaqoob, X. Heng, L. M. Lee, X. Cui, and C. Yang, "Full field phase imaging using a harmonically matched diffraction grating pair based homodyne quadrature interferometer," App. Phys. Lett. 90, 151123 (2007).

16. Z. Yaqoob, J. Wu, X. Cui, X. Heng, and C. Yang, "Harmonically-related diffraction gratings-based interferometer for quadrature phase measurements," Opt. Express 14, 8127-8137 (2006).

17. M. A. Choma, C. Yang, and J. A. Izatt, "Instantaneous quadrature low-coherence interferometry with $3 \times 3$ fiberoptics couplers," Opt. Lett. 28, 2162-2164 (2003) 
18. Z. Yaqoob, J. Fingler, X. Heng, and C. Yang, "Homodyne en face optical coherence tomography," Opt. Lett. 31, 1815-1817 (2006).

19. C. M. B. Cordeiro, L. Cescato, A. A. Freschi, and Lifeng Li, "Measurement of phase differences between the diffracted orders of deep relief gratings," Opt. Lett. 28, 683-685 (2003)

20. M. Pilu, A. Fitzgibbon, and R. Fisher, "Ellipse-specific direct least-square fitting," in Proceedings of IEEE International Conference on Image Processing (Lausanne, 1998), vol. 3, pp. 599-602.

21. D. G. Ghiglia and M. D. Pritt, Two-Dimensional Phase Unwrapping: Theory, Algorithms, and Software, Section 4.5, John Wiley \& Sons, 1998. 